\title{
Aptamer targeted therapy potentiates immune checkpoint blockade in triple- negative breast cancer
}

Simona Camorani ${ }^{1}$, Margherita Passariello ${ }^{2,3}$, Lisa Agnello ${ }^{1}$, Silvia Esposito ${ }^{3}$, Francesca Collina ${ }^{4}$, Monica Cantile ${ }^{4}$, Maurizio Di Bonito ${ }^{4}$, Ilya V. Ulasov ${ }^{5}$, Monica Fedele ${ }^{1}$, Antonella Zannetti ${ }^{6}$, Claudia De Lorenzo ${ }^{2,3^{*}}$ and Laura Cerchia ${ }^{1 *}$ (D)

Abstract: Background: Triple-negative breast cancer (TNBC) is a uniquely aggressive cancer with high rates of relapse due to resistance to chemotherapy. TNBC expresses higher levels of programmed cell death-ligand 1 (PDL1) compared to other breast cancers, providing the rationale for the recently approved immunotherapy with antiPD-L1 monoclonal antibodies (mAbs). A huge effort is dedicated to identify actionable biomarkers allowing for combination therapies with immune-checkpoint blockade. Platelet-derived growth factor receptor $\beta$ (PDGFR $\beta$ ) is highly expressed in invasive TNBC, both on tumor cells and tumor microenvironment. We recently proved that tumor growth and lung metastases are impaired in mouse models of human TNBC by a high efficacious PDGFR aptamer. Hence, we aimed at investigating the effectiveness of a novel combination treatment with the PDGFR $\beta$ aptamer and anti-PD-L1 mAbs in TNBC.

Methods: The targeting ability of the anti-human PDGFR $\beta$ aptamer toward the murine receptor was verified by streptavidin-biotin assays and confocal microscopy, and its inhibitory function by transwell migration assays. The anti-proliferative effects of the PDGFRß aptamer/anti-PD-L1 mAbs combination was assessed in human MDA-MB231 and murine 4T1 TNBC cells, both grown as monolayer or co-cultured with lymphocytes. Tumor cell lysis and cytokines secretion by lymphocytes were analyzed by LDH quantification and ELISA, respectively. Orthotopic 4T1 xenografts in syngeneic mice were used for dissecting the effect of aptamer/mAb combination on tumor growth, metastasis and lymphocytes infiltration. Ex vivo analyses through immunohistochemistry, RT-qPCR and immunoblotting were performed.

(Continued on next page)

\footnotetext{
*Correspondence: cladelor@unina.it; cerchia@unina.it;

laura.cerchia@ieos.cnr.it

${ }^{2}$ Department of Molecular Medicine and Medical Biotechnology, University of Naples "Federico II", Via Pansini 5, 80131 Naples, Italy

${ }^{1}$ Institute of Experimental Endocrinology and Oncology "Gaetano Salvatore",

CNR, Via S. Pansini 5, 80131 Naples, Italy

Full list of author information is available at the end of the article
}

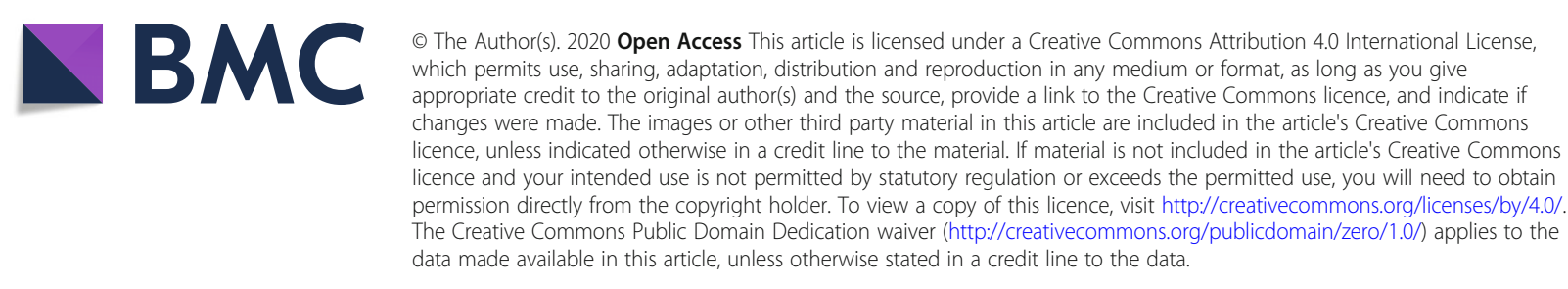




\begin{abstract}
(Continued from previous page)
Results: We show that the PDGFRß aptamer potentiates the anti-proliferative activity of anti-PD-L1 mAbs on both human and murine TNBC cells, according to its human/mouse cross-reactivity. Further, by binding to activated human and mouse lymphocytes, the aptamer enhances the anti-PD-L1 mAb-induced cytotoxicity of lymphocytes against tumor cells. Importantly, the aptamer heightens the antibody efficacy in inhibiting tumor growth and lung metastases in mice. It acts on both tumor cells, inhibiting Akt and ERK1/2 signaling pathways, and immune populations, increasing intratumoral CD8 + T cells and reducing FOXP3 + Treg cells.

Conclusion: Co-treatment of PDGFRß aptamer with anti-PD-L1 mAbs is a viable strategy, thus providing for the first time an evidence of the efficacy of PDGFRß/PD-L1 co-targeting combination therapy in TNBC.
\end{abstract}

Keywords: Aptamer, Antitumor immunity, PDGFRß, PD-L1 monoclonal antibody, TNBC, Tumor microenvironment, Metastases

\section{Background}

Triple-negative breast cancer (TNBC), accounting for $15-20 \%$ of breast cancers, is a heterogeneous group of tumors with highly metastatic behavior, poor prognosis and an urgent therapeutic need [1,2]. The lack of expression of oestrogen receptor, progesterone receptor and epidermal growth factor receptor 2 (HER2) makes TNBC difficult to treat leaving chemotherapy as the solely available option for most patients, both in early and advanced stages of the disease, despite its considerable side effects and limited success [3-5]. Nevertheless, thanks to of the continuous effort in searching molecularly targeted approaches for TNBC, the first targeted therapies have been recently approved. These consist in the PARP inhibitors Olaparib or Talazoparib [6], which is beneficial for BRCA-mutated patients but efficiently target TNBC cells regardless of the BRCA-status, and the anti-programmed cell death-ligand 1 (PD-L1) Atezolizumab plus nab-paclitaxel chemotherapy [7], which applies to patients with unresectable locally advanced or metastatic PD-L1-positive TNBCs.

The rationale for the latter regimen, representing the first immunotherapy to be approved for the treatment of breast cancer, stands on the significant role of the immune system in TNBC [8]. High PD-L1 expression and amplification of CD274 (encoding PD-L1) have been found in most TNBC [9] and, along with the presence of tumor-infiltrating lymphocytes (TILs), has been shown to influence TNBC prognosis [10]. Thus, by blocking the interaction of PD-L1, on tumor cells, with PD-1 and B7.1 receptors, on tumorinfiltrating T-cells and antigen-presenting cells, the antiPD-L1 monoclonal antibody (mAb) Atezolizumab causes a reduction of immunosuppressive signals within the tumor microenvironment (TME). This in turn causes the enhancement of $\mathrm{T}$ cell-mediated immunity against tumors [11]. Noteworthy, ongoing clinical studies are exploring combination approaches of various targeting agents together with anti-PD-1/PD-L1 mAbs aimed at maximizing the effectiveness of the treatment, especially for patients with metastatic TNBC, that have only modest response to immune checkpoint inhibitors as monotherapy [12, 13]. Among these, based on preclinical evidence of therapeutic synergy, clinical trials for TNBC treatment are currently underway, or are in recruitment status, by combining antiPD-1/PD-L1 mAbs with small-molecules inhibitors of receptor tyrosine kinases (RTKs). Some examples are the inhibitors of Axl (NCT03184558), VEGFR (NCT03394287, NCT03797326) and c-Kit (NCT03855358). Furthermore, there is a rapid increase of the number of studies showing the efficacy of co-blocking PD-1, or its ligand, and RTKs in various human cancers [14-17], including TNBC [18].

Platelet-derived growth factor receptor $\beta$ (PDGFR $\beta$ ) is a transmembrane RTK expressed on endothelial and perivascular cells, where it plays an important role in wound healing and tissue repair, inflammation and angiogenesis [19]. It is well known that overexpression of PDGFR $\beta$ on endothelial cells and tumor-associated stromal cells surface occurs in different human cancers, where the receptor establishes complex signaling pathways inducing angiogenesis and tumor progression [20-22]. Moreover, PDGFR $\beta$ expression has been shown as a unique feature of tumor cells characterized by a mesenchymal/stem and poorly differentiated phenotype, and it correlates with aggressiveness and resistance to therapy in multiple tumor types [23-30]. Recent findings prove that PDGFR $\beta$ is expressed on the surface of tumor cells belonging to a subgroup of mesenchymal TNBC with invasive and stem-like phenotype and contributes to drive the metastatic potential [31] and vasculogenic mimicry [31, 32] of these tumors.

In searching efficacious strategies to target PDGFR $\beta$ positive TNBC in alternative to PDGFR $\beta$ tyrosine kinase inhibitors, which showed limited clinical activity in TNBC as single agents and severe side effects [33, 34], we recently tested the Gint4.T nuclease-resistant RNA aptamer, which we previously validated as a high affinity ligand/inhibitor of PDGFR $\beta$ in glioblastoma (GBM) [35, 36] and human bone marrow-derived mesenchymal stem cells (BM-MSCs) [22]. We found that Gint4.T is a potent theranostic agent in TNBC [31], as it efficiently 
detected lung metastases derived from TNBC cells and suppressed their formation when intravenously administrated in a mouse model [31].

The aim of the present study was to investigate the effectiveness of the combination of the Gint4.T aptamer with anti-PD-L1 antibodies in TNBC since there are no studies including a combined inhibition of both PDGFR $\beta$ and PD-1/PD-L1 interaction in the treatment of these tumors. The inhibitory effects of combination of Gint4.T with anti-PD-L1 monoclonal antibodies on tumor cells growth, in monolayer and in co-cultures with lymphocytes, were tested in both human and mouse TNBC cell models. Importantly, we show that the PDGFR $\beta$ aptamer augments antitumor immunity and potentiates anti-PD-L1 antibody inhibitory effects on tumor growth and lung metastases formation in $4 \mathrm{~T} 1 \mathrm{TNBC}$ orthotopic mouse model.

\section{Methods}

\section{Cell cultures}

Growth conditions for human breast cancer MDA-MB231 and BT-474 cell lines, and murine NIH3T3 fibroblasts (American Type Culture Collection, ATCC, Manassas, VA) were previously reported [37]. The murine TNBC 4 T1 cells (ATCC) were grown in Roswell Park Memorial Institute-1640 medium (RPMI-1640, SigmaAldrich, Milan, Italy) supplemented with $10 \%$ heatinactivated fetal bovine serum (FBS, Sigma-Aldrich), in $95 \%$ air $/ 5 \% \mathrm{CO} 2$ atmosphere at $37^{\circ} \mathrm{C}$.

Human peripheral blood mononuclear cells (hPBMCs) were isolated and grown as previously described [38, 39]. Mouse lymphocytes were isolated from mouse spleen and grown in R10 medium consisting of RPMI-1640 medium, supplemented with $10 \%$ heat-inactivated FBS, $50 \mathrm{U} / \mathrm{ml}$ penicillin, $50 \mu \mathrm{g} / \mathrm{ml}$ streptomycin, $2 \mathrm{nML}$-glutamine, $10 \mathrm{mM}$ HEPES and $50 \mathrm{mM} \beta$-mercaptoethanol.

\section{Aptamers and monoclonal antibodies}

The sequences of the 2'Fluoro-pyrimidines (2'F-Py) RNA PDGFR $\beta$ Gint4.T and scrambled (Scr) aptamer, used as negative control, were previously reported [22]. Unlabeled and FAM-labeled aptamers were synthesized by TriLink Biotechnologies (San Diego, CA, USA). 5'-biotinylated Gint4.T and Scr were synthesized by LGC Biosearch Technologies (Risskov Denmark). The handling protocols for aptamers, prior to each treatment, were previously described [31].

Anti-human PD-L1 10_12 mAb, anti-mouse PD-L1 mAb (clone 10F.9G2, BioXcell) and unrelated IgG, used as negative control, were previously reported [40].

\section{Binding of Gint4.T aptamer to PDGFR $\beta$-positive murine} cells

\section{Binding affinity (Kd value) calculation}

Binding of Gint4.T to $4 \mathrm{~T} 1$ cells was assessed by streptavidin-biotin-based assay, as previously described [41]. Briefly, 4 T1 cells $\left(2.0 \times 10^{4}\right.$ cells/well in clear round bottom 96-well plate) were incubated for $10 \mathrm{~min}$ at room temperature (RT) with increasing concentrations of $5^{\prime}$ biotinylated Gint4.T or Scr aptamers $(10 \mathrm{nM}, 20 \mathrm{nM}, 50$ $\mathrm{nM}, 100 \mathrm{nM}, 200 \mathrm{nM}$ and $500 \mathrm{nM}$ ), diluted in the binding buffer (BB) consisting of BlockAid ${ }^{\mathrm{TM}}$ blocking solution (Invitrogen, Carlsbad, CA, USA) with $1 \mathrm{mg} / \mathrm{ml}$ yeast tRNA and $1 \mathrm{mg} / \mathrm{ml}$ ultrapure ${ }^{\mathrm{Tm}}$ salmon sperm DNA (Invitrogen), as nonspecific competitors. The binding affinity (Kd value) was calculated as previously reported [41], by using Scr to determine the nonspecific binding.

\section{Confocal microscopy}

To visualize Gint4.T on the surface of PDGFR $\beta$-positive murine cells, $4 \mathrm{~T} 1$ and NIH3T3 $\left(1.0 \times 10^{5}\right.$ cells/well in 24-well), previously seeded on a coverslip for $24 \mathrm{~h}$, were incubated with FAM-labeled Gint4.T or FAM-labeled Scr (500 nM-final concentration in BB) for $10 \mathrm{~min}$ at RT. PDGFR $\beta$-negative BT-474 cells were treated in the same condition and used as negative control. After three washes in Dulbecco's phosphate-buffered saline (DPBS), cells were fixed with $4 \%$ paraformaldehyde in DPBS for 20 min, washed three times in DPBS and incubated with $1.5 \mu \mathrm{M}$ 4',6-Diamidino- 2-phenylindole (DAPI, D9542, Sigma-Aldrich). Finally, coverslips were mounted with glycerol/DPBS. The fluorescence images were taken under a Zeiss LSM 700 META confocal microscopy equipped with a Plan-Apochromat 63x/1.4 Oil DIC objective.

\section{Inhibition of murine cells migration by Gint4.T aptamer} 4 T1 and NIH3T3 cells were serum starved overnight in the presence of $500 \mathrm{nM}$ Gint4.T or Scr. Then, cells (4 T1, $5 \times 10^{4} /$ well; NIH3T3, $2 \times 10^{5} /$ well) were seeded into the upper chamber of a 24-well transwell (Transwell filters $8 \mu \mathrm{m}$ pore size; Corning Incorporate, Corning, NY) in the presence of $500 \mathrm{nM}$ Gint4.T or Scr and exposed to medium containing PDGF-BB (50 ng/ml, R\&D Systems, Minneapolis, MN), as inducer of migration. After incubation for $24 \mathrm{~h}$ at $37^{\circ} \mathrm{C}$ in a humidified incubator in $5 \% \mathrm{CO} 2$, the migrated cells were visualized by staining with $0.1 \%$ crystal violet in $25 \%$ methanol and photographed. Stained cells were lysed in 1\% sodium dodecyl sulfate and absorbance at $595 \mathrm{~nm}$ was measured on a microplate reader.

\section{PDGF-BB stimulation of murine cells}

NIH3T3 cells $\left(1.5 \times 10^{5}\right.$ cells/well in 6 -well $)$ were mocktreated or serum-starved for $18 \mathrm{~h}$ and then left untreated 
or stimulated for $10 \mathrm{~min}$ with $20 \mathrm{ng} / \mathrm{mL}$ PDGF-BB. Cell lysates preparation and immunoblotting analyses with anti-phospho-PDGFR $\beta$ (Tyr771, indicated as p-PDGF $\mathrm{R} \beta$ ) (Cell Signaling Technology Inc.) primary antibodies were performed as previously reported [42] by using anti-vinculin (N-19) (Santa Cruz Biotechnology, Santa Cruz, CA) as loading control. Blots shown are representative of at least three independent experiments.

\section{Cell growth inhibition by Gint4.T aptamer/anti-PDL1 mAb combined treatment}

MDA-MB-231 $\left(5.0 \times 10^{3}\right.$ cells/well $)$ and 4 T1 $\left(3.0 \times 10^{3}\right.$ cells/well) were plated in 96-well and, after $16 \mathrm{~h}$ at $37^{\circ} \mathrm{C}$, were either untreated or treated for $96 \mathrm{~h}$ with $200 \mathrm{nM}$ Gint4.T and $100 \mathrm{nM}$ human anti-PD-L1 10_12 (MDAMB-231) or murine anti-mPD-L1 (4T1), used alone or in combination. Unrelated IgG $(100 \mathrm{nM})$ and Scr $(200$ $\mathrm{nM})$ were used as negative controls. The treatment with the aptamers was renewed after $72 \mathrm{~h}$. Cell counts were measured by the trypan blue exclusion test.

\section{Effects of combinatorial treatments on co-cultures of tumor cells and lymphocytes}

MDA-MB-231 $\left(1.5 \times 10^{4}\right.$ cells/well $)$ or $4 \mathrm{~T} 1\left(1.5 \times 10^{4}\right.$ cells/well) cells, previously seeded in 96-well flat-bottom plates for $16 \mathrm{~h}$ at $37^{\circ} \mathrm{C}$, were co-cultured with human or murine lymphocytes, respectively, at effector:target cells ratio 10:1, in the absence or presence of $200 \mathrm{nM}$ Gint4.T or $100 \mathrm{nM}$ anti-PD-L1 10_12 (human setting) or antimPD-L1 (mouse setting), used alone or in combination. Unrelated IgG (100 nM) and Scr $(200 \mathrm{nM})$ were used as negative controls. After $24 \mathrm{~h}$ incubation at $37^{\circ} \mathrm{C}$ in a humidified incubator in 5\% CO2, lymphocytes were removed and adherent cells were washed and counted by the trypan blue exclusion test.

For determination of tumor cell lysis, the release of lactate dehydrogenase (LDH) in the cellular co-culture supernatants was measured by a LDH detection kit (Thermofisher Scientific, Meridian Rd., Rockoford, IL, USA), as previously described [38, 39].

The concentration of interleukin-2 (IL-2) or interferon gamma (IFN- $\gamma$ ) cytokines secreted in the cellular cocultures supernatant was measured by ELISA assays (DuoSet ELISA, R\&D Systems, Minneapolis, MN, USA), as previously described $[38,39]$.

\section{Binding of Gint4.T aptamer to human and mouse lymphocytes}

Binding of 5'-biotinylated Gint4.T to human or mouse activated lymphocytes was assessed as previously described [38] by using increasing concentrations $(50 \mathrm{nM}$, $100 \mathrm{nM}$ and $200 \mathrm{nM}$ ) of 5 '-biotinylated Gint4.T or Scr aptamers.

\section{In vivo experiments}

4 T1 cells $\left(3 \times 10^{4}\right)$ were re-suspended in $0.1 \mathrm{ml}$ of $1: 1$ mix of physiological saline and Matrigel (BD Biosciences, Franklin Lakes, NJ) and orthotopically injected into the mammary fat pads of five-week-old Female Balb/c mice, which weighed about 20-22 g (Charles River, Milan, Italy). Once tumors became approximately $150 \mathrm{~mm}^{3}$ [volume $=0.5 \times$ long diameter $\left.\times(\text { short diameter })^{2}\right]$, mice were randomized into four groups (five mice for each group): Ctrl (1400 pmol Scr/intravenous injection, at day 0, 2, 4, 7 and 9); Gint4.T aptamer (1400 pmol Gint4.T/ intravenous injection, at day 0, 2, 4, 7 and 9); anti-mPDL1 $(200 \mu \mathrm{g} /$ intraperitoneal injection, at day 0,4 and 9$)$; Gint4.T plus anti-mPD-L1 (1400 pmol Gint4.T/intravenous injection at day $0,2,4,7$ and 9 plus $200 \mu \mathrm{g}$ anti$\mathrm{mPD}-\mathrm{L} 1 /$ intraperitoneal injection, at day 0,4 and 9). The long and short diameters of the tumors were measured using slide calipers up to day 11 (2 days after the last treatment) and the body weight was also measured. At day 11, mice were euthanized. Treatment schedule is schematized in Fig. 4a.

\section{Ex vivo analyses}

After sacrificing mice, tumors from each animal were excised and cut into two pieces for sample processing: one piece was stored in 10\% neutral buffered formalin for immunohistochemistry analyses, and the other piece was frozen, using liquid nitrogen, for RNA extraction and reverse transcription quantitative polymerase chain reaction (RT-qPCR) and protein lysis for immunoblotting analyses. Lung from each animal were harvested and stored in $10 \%$ neutral buffered formalin for immunohistochemistry analyses.

\section{$R T-q P C R$}

RNA extraction and RT-qPCR were performed as previously described [31] on tumors from four animals per group. Primers used were: IL-2, Fwd 5'-TTGTCGTCCT TGTCAACAGC-3', Rev. 5' - CTGGGGAGTTTCAGGT TCCT-3'; IFN- $\gamma$, Fwd, 5' -AGCGGCTGACTGAACTCA GATTGTAG-3', Rev. 5'-GTCACAGTTTTCAGCTGT ATAGGG-3'. The following primers were used for internal control: Glucose-6-phosphate dehydrogenase, Fwd 5'-TTATCATCATGGGTGCATCG-3', Rev. 5'-GCATAGCCCACAATGAAGGT-3'.

\section{Immunoblotting analyses on tumor lysates}

Tumor lysates preparation and immunoblotting analyses were performed as previously reported [42]. Filters were probed with the indicated primary antibodies: antiPDGFR $\beta$, anti-phospho-44/42 MAPK (extracellular signal-regulated kinase $1 / 2$, ERK $1 / 2$, indicated as $p$ ERK1/2), anti-phospho-Akt (Ser473, indicated as p-Akt), anti-Akt (Cell Signaling Technology Inc.), anti-PD-L1/ 
CD274 (Proteintech Group, Inc.), anti-ERK1 (C-16) and anti-vinculin (N-19) (Santa Cruz Biotechnology, Santa Cruz, CA). Blots shown are representative of at least three independent experiments.

\section{Immunohistochemistry}

Formalin-fixed tumors and lungs were paraffinembedded and sectioned $(4 \mu \mathrm{m})$ and three samples per group were stained with hematoxylin and eosin (H\&E) or immunostained as reported [31]. The primary antibodies used were: anti-CD8 alpha antibody (clone D4W2Z, \#98941S, dilution $1: 500,1 \mathrm{~h} 4{ }^{\circ} \mathrm{C}$ incubation, Cell Signaling Technology Inc.); anti-FoxP3 (clone D608R, \#12653S, dilution 1:150, $1 \mathrm{~h} 4{ }^{\circ} \mathrm{C}$ incubation, Cell Signaling); anti-Granzyme B (GRZB ab4059; dilution 1: 150 diluted, $4^{\circ} \mathrm{C}$ RT, Abcam, Cambridge, MA); anti-Ki67 (dilution $1: 75$, overnight $4{ }^{\circ} \mathrm{C}$ incubation, Invitrogen); anti-PDGFR $\beta$ (diluition 1:50, $1 \mathrm{~h} 4{ }^{\circ} \mathrm{C}$ incubation, Cell signaling Technology Inc.); anti-PD-L1 (diluition 1:50, 1 h $4{ }^{\circ} \mathrm{C}$ incubation, Proteintech Group).

Results were interpreted using Olympus BX43 light microscope (Olympus, Center Valley, PA). Each slide was reviewed blinded and, to ensure accuracy, the number of metastases and of Ki-67, CD8, GRZB and Foxp3positive cells was determined by two independent counts.

\section{Statistical analysis}

Statistical values were defined using GraphPad Prism version 8.00 by unpaired $t$-test (two variables) or oneway ANOVA followed by Tukey's multiple comparison test (more than two variables). $P$ value $<0.05$ was considered significant for all analyses.

\section{Results}

\section{The PDGFR $\beta$ aptamer enhances the anti-proliferative} activity of anti-PD-L1 mAb on human TNBC cells

As both PDGFR $\beta$ and PD-L1 are crucial targets for TNBC treatments [31, 43, 44], we tested whether their co-inhibition would enhance TNBC cells killing. To this aim, we used an aptamer and a mAb that we previously validated as high affinity binders and inhibitors of PDGFR $\beta$ [31] and PD-L1 [40, 45], respectively, in different tumor types including TNBC. Specifically, Gint4.T is a 2'F-Py RNA aptamer that binds to the extracellular domain of PDGFR $\beta$ and inhibits receptor activation and downstream phosphatidyl-3-kinase (PI3K)/Akt and ERK1/2 pathways [22, 31, 35], thus affecting growth, migration and invasion of mesenchymal TNBC cell lines [31]. On the other side, the high affinity human anti-PDL1 (10_12) mAb [38, 46] not only interferes in the PD-L1/PD-1 interaction but also inhibits the immune- independent PD-L1 positive TNBC cell growth by affecting the intracellular MAPK pathway [40].

First, we analyzed the effects of Gint4.T aptamer and 10_12 mAb used in combination, along with single agents, on the growth of human TNBC MDA-MB-231 cells, expressing high levels of both PDGFR $\beta$ [31, 37] and PD-L1 [40], further enhanced by previous cell exposure to cisplatin and doxorubicin, two chemotherapeutics used in clinic for TNBC [41]. Based on our previous dose and time-response analyses in MDA-MB231 cells for the aptamer [31] and antibody [40], we used them at a concentration of 200 and $100 \mathrm{nM}$, respectively, prolonging the incubation up to $96 \mathrm{~h}$. Indeed, under these experimental conditions the inhibitory effects of both the aptamer and antibody is not so strong as it is at higher concentration and/or longer incubation time [31, 40], thus allowing to appreciate a potential enhancement of their effects in combination. As shown in Fig. 1a, the combined treatment led to a significantly stronger reduction of cell growth (50\% inhibition), compared to either the single anti-PD-L1 antibody (37\% inhibition) or anti-PDGFR $\beta$ aptamer (25\% inhibition). As expected, no effects were observed in the presence of an unrelated IgG antibody and a scrambled aptamer (Scr), used as negative controls. Next, in order to assess whether the aptamer-mediated inhibition of PDGFR $\beta$ enhances the inhibitory effects on cell growth exerted by $10 \_12 \mathrm{mAb}$ in the context of PD-1/PD-L1 interaction between TNBC cells and infiltrating lymphocytes [38, 40], we tested the aptamer plus mAb on co-cultures of MDAMB-231 cells with human lymphocytes by using hPBMCs (effector:target ratio 10:1) (Fig. 1b-e). Noteworthy, following $24 \mathrm{~h}$-treatment, Gint4.T even though not exerting itself an appreciable reduction of cell growth when used as single agent, it caused a significant increase of the 10_12 mAb inhibitory effect on cell growth, that reached about $50 \%$ inhibition with respect to $28 \%$ inhibition observed when the mAb was used alone (Fig. 1b). Further, the co-treatment of the cells with Gint4.T aptamer and 10_12 mAb had a more potent cytotoxic effect, as assessed by higher levels of $\mathrm{LDH}$ released by tumor cells co-cultured with lymphocytes, than that observed with the sole anti-PD-L1 antibody (Fig. 1c). Moreover, a significant increase of IL-2 (Fig. 1d) and IFN- $\gamma$ (Fig. 1e) secretion was detected when MDA-MB-231 cells were co-cultured with lymphocytes and treated with 10_12 with respect to untreated cocultured cells, with IL-2 secretion that was further increased by the combined Gint4.T/10_12 treatment (Fig. $1 d)$. Accordingly to previous findings showing that PDGFR $\beta$ is expressed on T cells and PDGF-BB, its primary ligand, potently regulates $\mathrm{T}$ cells function [47], we found that Gint4.T binds to the receptor on the surface of activated lymphocytes, as measured by cell-ELISA 
A

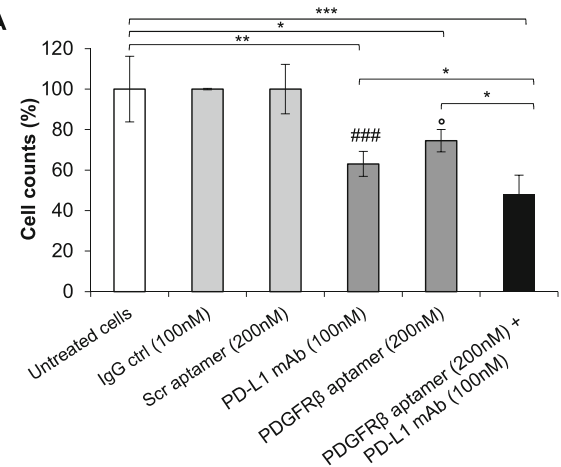

B

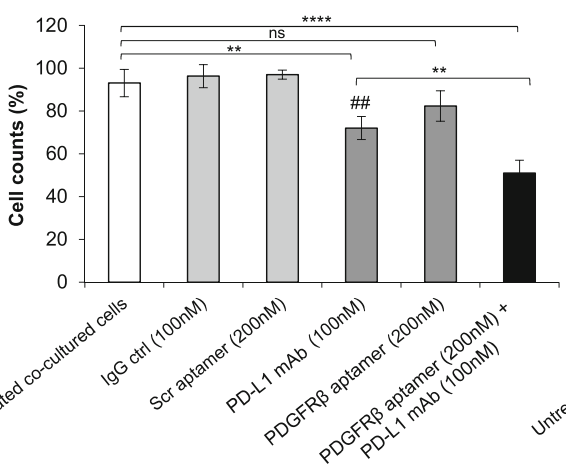

D

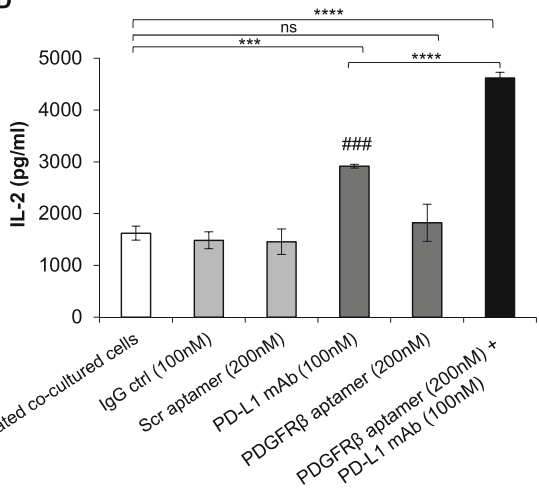

C

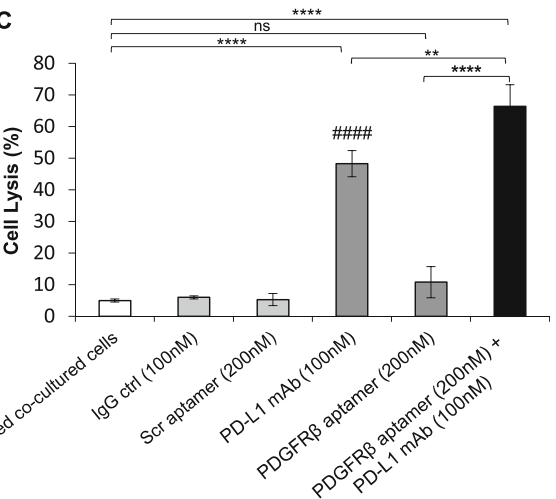

$E$

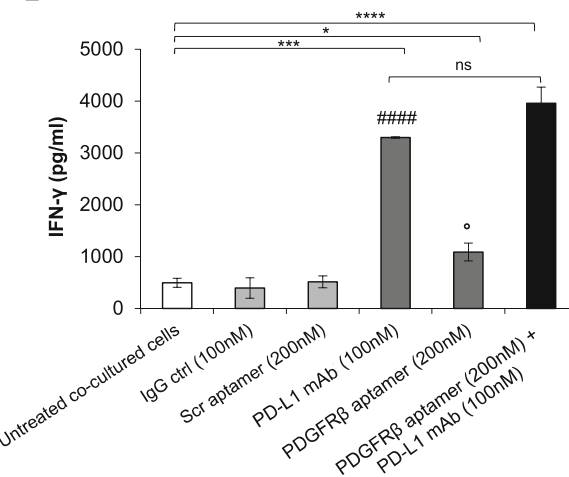

Fig. 1 Effects of combinatorial treatments on human MDA-MB-231 cells in the absence or presence of lymphocytes. a Cell growth inhibition of MDA-MB-231 cells, untreated or treated with the anti-PD-L1 (10_12) mAb or the PDGFRß aptamer, used alone (dark grey bars) or in combination (black bars), for $96 \mathrm{~h}$ at $37^{\circ} \mathrm{C}$ at the indicated concentrations. Untreated cells (white bars) or cells treated with an unrelated lgG or Scr (light grey bars) were used as negative controls. Data were expressed as percentage of viable treated cells with respect to untreated cells. b-e MDA-MB-231 cells were co-cultured with human lymphocytes and left untreated or treated for $24 \mathrm{~h}$, as indicated. $\mathbf{b}$ Cell counts were expressed as percentage of viable treated cells with respect to untreated co-cultured cells. c Cell lysis was expressed as measure of LDH release and reported as percentage of lysis of treated cells with respect to the untreated cells, used as a control. $\mathbf{d} \mathrm{IL}-2$ and $\mathbf{e} \mathrm{IFN}-\gamma$ secretion levels were measured by

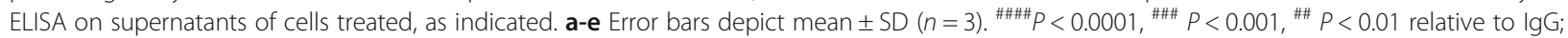
${ }^{\circ} P<0.05$ relative to Scr; ${ }^{* * * *} P<0.0001,{ }^{* * *} P<0.001,{ }^{* *} P<0.01,{ }^{*} P<0.05$; one-way ANOVA followed by Tukey's multiple comparison test

assays (Figure S1A), thus suggesting that it may modulate cytokines secretion by lymphocytes.

\section{The PDGFR $\beta$ aptamer binds to and inhibits the murine receptor}

In order to test the promising aptamer/mAb combination treatment in vivo, by exploiting a fully immunocompetent murine preclinical syngeneic model, first we checked the capability of Gint4.T aptamer, selected to recognize the human receptor [35], to bind to PDGFR $\beta$-positive $4 \mathrm{~T} 1$ cells, an established mouse model for aggressive TNBC cells [48, 49]. Even if some species specificity has been shown in aptamers recognition ability [50], we hypothesized a high affinity binding for Gint4.T aptamer to murine PDGFR $\beta$ because it shares about $80 \%$ sequence identity in the 
extracellular region with the human receptor (Figure S2A) and, accordingly, the recombinant human PDGF-BB ligand, efficiently stimulates the murine receptor expressed on PDGFR $\beta$-positive NIH3T3 cells (Figure S2B). As expected, Gint4.T efficiently binds to $4 \mathrm{~T} 1$ cells with a $\mathrm{Kd}$ value of $76.76 \pm 14.69 \mathrm{nM}$ (Fig. 2a), which was comparable to that observed on human MDA-MB-231 cells $(57.48 \pm 7.8 \mathrm{nM})$ [31]. Confocal microscopy analyses on both $4 \mathrm{~T} 1$ and NIH3T3 cells confirmed the ability of FAM-labeled
Gint4.T to decorate the surface of murine cells (Fig. $2 b)$. Incubation of Gint4.T on PDGFR $\beta$-negative BT-4 T4 breast cancer cells [31] and of Scr on NIH3T3 cells (Fig. 2b) were used as negative controls in parallel analyses.

Next, given the driving role of PDGFR $\beta$ on cell motility and the ability of Gint4.T to hamper migration and invasion of human cancer cells [22,31,35], to confirm its effect on murine receptor upon binding, we proved that the aptamer strongly reduces the PDGF-BB-stimulated migration of $4 \mathrm{~T} 1$

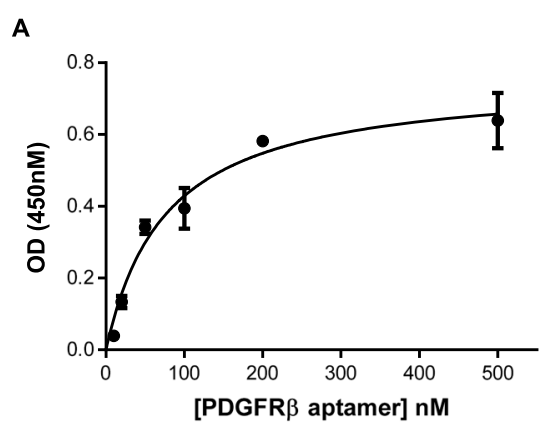

B

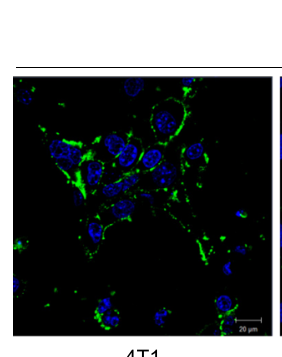

(PDGFRß-positive)
FAM-PDGFR $\beta$ aptamer DAPI

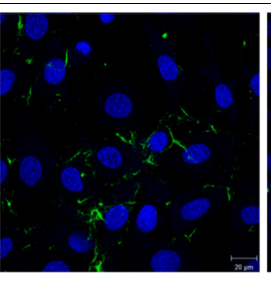

$\mathrm{NIH} 3 \mathrm{~T} 3$

(PDGFRß-positive)

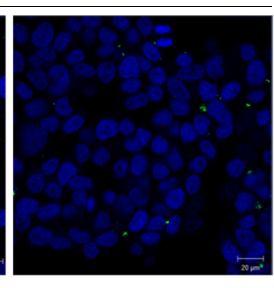

BT-474 (PDGFRß-negative)

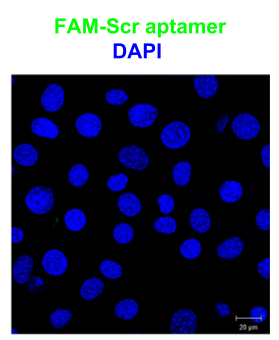

NIH3T3

(PDGFRß-positive)

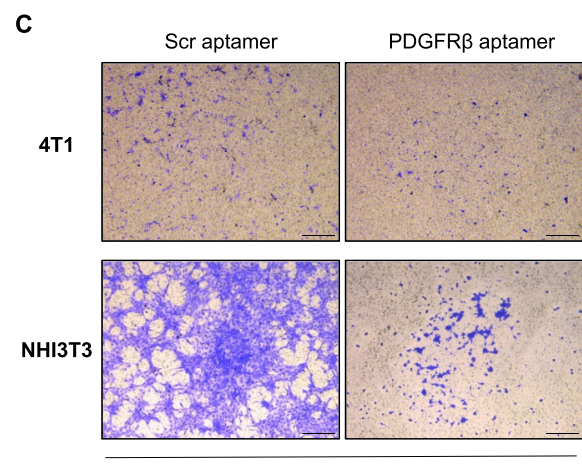

PDGF-BB

Fig. 2 Gint4.T aptamer specifically binds to and inhibits migration of PDGFRß-positive murine cells. a Binding curve of 5'-biotinylated PDGFR Gint4.T aptamer to 4 T1 cells for calculation of the apparent Kd of aptamer-cell interaction. The background binding value for 5'-biotinylated Scr sequence was subtracted from each data point. Data shown are mean \pm SD $(n=3)$. b Representative confocal images of 4 T1, NIH3T3 and BT-474 cells incubated with FAM-Gint4.T or FAM-Scr, as indicated. FAM-aptamers and nuclei are visualized in green and blue, respectively. All digital images were captured at the same setting to allow for direct comparison of staining patterns. Magnification $63 \times$, scale bar $=20 \mu \mathrm{m}$. $\mathbf{c}$ Left, $4 \mathrm{T1}$ and NIH3T3 cell migration toward PDGF-BB was analyzed by transwell migration assay in the presence of Gint4.T or Scr. Photographs of a representative experiment are shown. Magnification $5 \times$, scale bar $=500 \mu \mathrm{m}$. Right, data were presented as percentage of migrated cells in the presence of Gint4.T with respect to cells treated with Scr. Bars depict mean \pm SD $(n=3) .{ }^{* * *} P<0.001{ }^{* * *} P<0.01$ relative to Scr; unpaired t-test 
and NIH3T3 cells, reaching about 45 and 70\% inhibition, respectively, compared to the Scr aptamer (Fig. 2c).

Overall, these results, showing mouse-human crossreactivity of Gint4.T, make the aptamer a valuable tool for preclinical therapeutic and/or diagnostic evaluation studies in murine environments.

\section{Efficacy of PDGFR $\beta$ and PD-L1 co-blockade on murine TNBC cells}

Before moving to the in vivo experiments, we first verified the effectiveness of PDGFR $\beta$ and PD-L1 combined inhibition on murine $4 \mathrm{~T} 1$ cells in vitro. Considering the low cross-reactivity of 10_12 mAb for the murine PD-L1 [40], we used a validated anti-mouse PD-L1 mAb (clone 10F.9G2, BioXcell) for PD-L1 blocking [51]. Under the same treatment conditions as for MDA-MB-231 cells, which gave rise to just a slight and not even significant inhibition of $4 \mathrm{~T} 1$ cell growth in monolayer by anti-mPDL1, the combination of Gint4.T and anti-mPD-L1 had a greater effect, which was significant compared to the single antibody treatment while there was a trend toward a greater beneficial compared with the aptamer alone that had already a significant effect as a single agent (Fig. 3a). According to the results obtained with human cells, the potentiated effects of the combinatorial approach over the use of single agents were also observed in co-cultures of tumor cells with mouse lymphocytes on both cell growth (Fig. 3b) and lysis (LDH release) (Fig. 3c). Finally, the cotreatment of the cells with Gint4.T and anti-mPD-L1 $\mathrm{mAb}$ resulted in an increased secretion of both IL- 2 and IFN- $\gamma$ compared with either compound alone (Fig. 3d). Interestingly, as previously observed on the human cells, Gint4.T binds to activated mouse lymphocytes (Figure S1B) which is consistent with the observed cytotoxic effects (Fig. 3b and c) caused by the aptamer in co-cultures experiments when used as single agent, and with its ability to potentiate the antibody effect in the combined treatment (Fig. 3d and e).

\section{Targeting PDGFR $\beta$ in vivo potentiates anti-PD-L1 efficacy modifying the tumor microenvironment}

Next, in vivo therapeutic effects of the combinatorial anti-PDGFR $\beta$ and anti-PD-L1 treatment was evaluated by using orthotopic $4 \mathrm{~T} 1$ xenografts in syngeneic BALB/ c model, representing the TNBC subtype $[48,52,53]$.

Treatments were initiated at 14 days after cell inoculation, when tumor mean volume was $\sim 150 \mathrm{~mm}^{3}$, and tumor growth was monitored by a caliper measuring tumor size for further 12 days. The anti-PDGFR $\beta$ aptamer (at a dose of $1400 \mathrm{pmol}, 0.74 \mathrm{mg} / \mathrm{kg}$ mean bodyweight, at day $0,2,4,7$ and 9) and anti-mPD-L1 mAb (at a dose of $10 \mathrm{mg} / \mathrm{kg}$ mean body-weight, at day 0,4 and 9) were administered intravenously or intraperitoneally, respectively, alone and in combination, according to the reference dosage of the aptamer [31] and antibody $[40,51]$, as single agents, for mice treatment and that of Atezolizumab and other immunomodulators in clinic [54]. Mice treated with scrambled aptamer served as control group (Ctrl). The results showed a significant inhibitory effect on tumor growth in mice treated with either Gint4.T or anti-mPD-L1, both if administrated alone or in combination, with respect to control group, which formed faster-growing tumors (Fig. 4a). Interestingly, the aptamer significantly enhanced the efficacy of the antibody in the combined treatments $(1.87 \pm 1.08$ vs $3.54 \pm 0.66$, Gint4.T plus anti-mPD-L1 vs anti-mPD-L1, $P=0.016)$. The treatments were well tolerated in vivo, with no significant loss in mice body weight over the entire treatment period (Fig. 4b). Also, the tumor proliferative potential was drastically hampered by Gint4.T plus anti-mPD-L1 treatment compared with either single agent as assessed by immunohistochemical staining for Ki-67-positive cells in the tumors (Fig. 4c). Next, accordingly to the strong inhibition of tumor growth, immunoblotting analyses performed on lysates from treated tumors showed a marked inhibition of Akt and ERK1/2 pathways, downstream of both PDGFR $\beta$ [22, 31, 35] and oncogenic PD-L1 $[40,55]$, in the tumors from mice treated with the aptamer, antibody and their combination (Fig. 4d). Interestingly, the combined treatment decreased the extent of Akt and ERK1/2 phosphorylation more efficiently than the treatment with aptamer or anti-PD-L1 mAb alone, showing a significant or a decreasing trend, respectively (Fig. 4d). No changes of PDGFR $\beta$ and PD-L1 expression were observed upon treatments as assessed both by immunoblotting (Fig. 4e) and immunohistochemistry (Figure S3).

Next, immunohistochemical analyses were performed on tumors from treated groups and compared with those from the Ctrl group. First, H\&E staining revealed the high extent of lymphocytes infiltration in the tumors, which is expected for high aggressive TNBC (Fig. 5a). Then, we stained tumors with antibodies specific for anti-CD8 and FoxP3-expressing regulatory $\mathrm{T}$ (Treg) cells, and GRZB, established indicators of antitumor activity [56]. As shown, we found an increase of both intratumoral CD8+ T cells (Fig. 5b and e) and GRZB positive staining (Fig. $5 \mathrm{c}$ and e) following Gint4.T plus anti-mPD-L1-treatment with respect to anti-PD-L1 or PDGFR $\beta$ aptamer single treatments. Conversely, a reduction in the number of Treg cells, as assessed by quantification staining of FoxP3, was observed when using either PDGFR $\beta$ Gint4.T aptamer alone or combining the Gint4.T treatment with the anti-PD-L1 compared to anti-PD-L1 alone (Fig. 5e). This is consistent with the findings that depletion of Treg cells is effective in evoking antitumor immune responses by promoting tumor infiltration of $\mathrm{CD} 8+\mathrm{T}$ cells with tumor-specific 


\section{A}

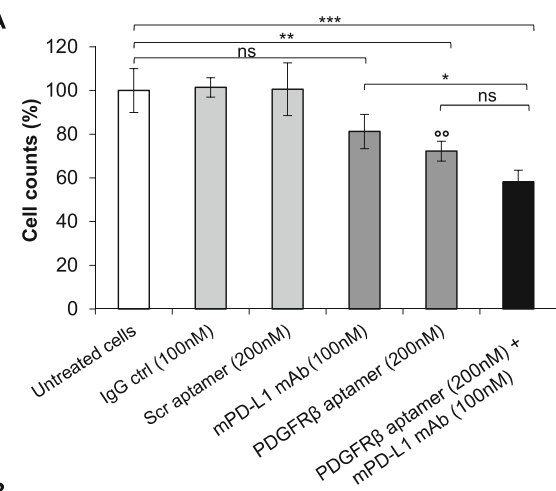

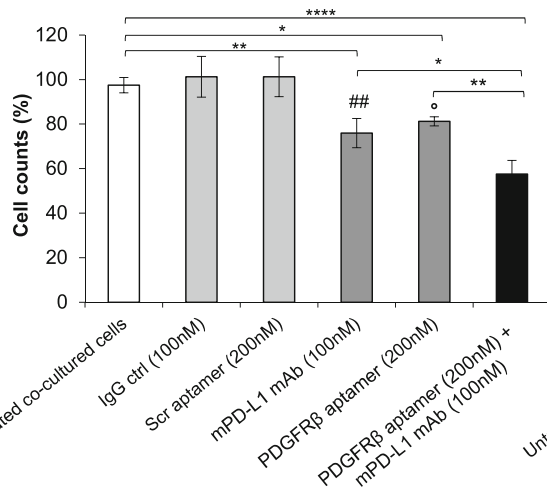

D
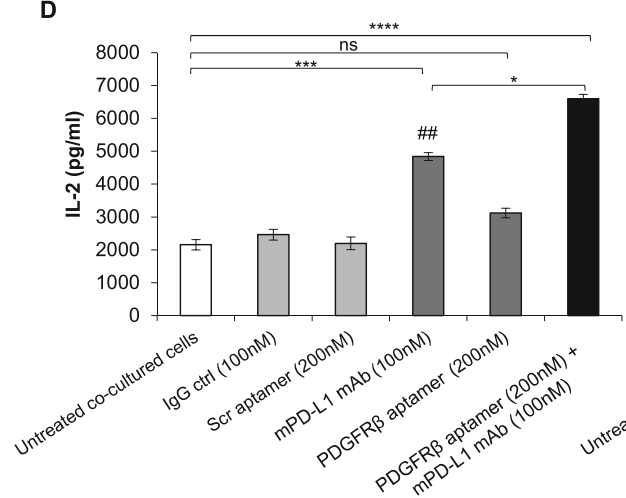

C

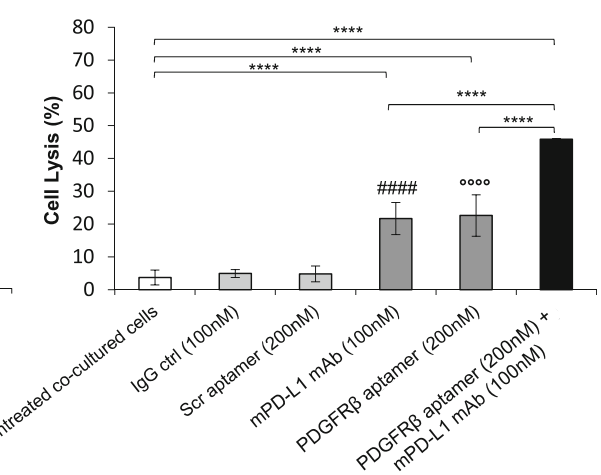

E

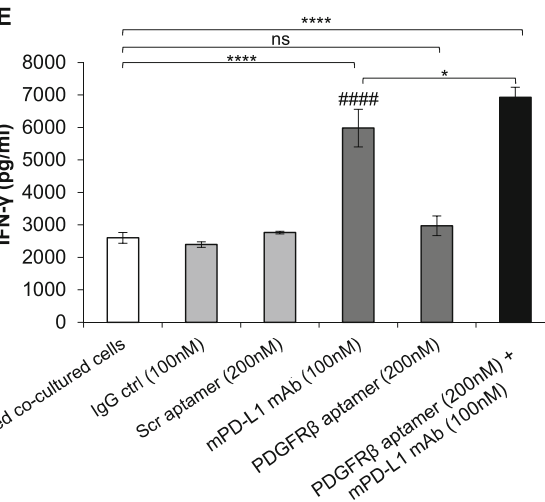

Fig. 3 Effects of combinatorial treatments on murine $4 \mathrm{~T} 1$ cells in the absence or presence of lymphocytes. a Cell growth inhibition of $4 \mathrm{~T} 1$ cells, untreated or treated with the anti-mPD-L1 mAb or the PDGFRß aptamer, used alone (dark grey bars) or in combination (black bars), for $96 \mathrm{~h}$ at $37^{\circ} \mathrm{C}$ at the indicated concentrations. Untreated cells (white bars) or cells treated with an unrelated lgG or Scr (light grey bars) were used as negative controls. Data were expressed as percentage of viable treated cells with respect to untreated cells. b-e $4 \mathrm{~T} 1$ cells were co-cultured with mouse lymphocytes and treated for $24 \mathrm{~h}$, as indicated. $\mathbf{b}$ Cell counts were expressed as percentage of viable treated cells with respect to untreated co-cultured cells. c Cell lysis was measured by LDH release in the medium after incubation with the indicated compounds. $\mathbf{d} I L-2$ and

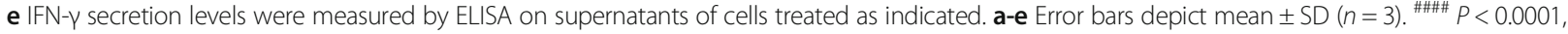
$\# \#<0.01$ relative to lgG; ${ }^{\circ 000} P<0.0001,{ }^{\circ 0} P<0.01,{ }^{\circ} P<0.05$ relative to Scr; ${ }^{* * * *} P<0.0001,{ }^{* * * *} P<0.001,{ }^{* *} P<0.01,{ }^{*} P<0.05$; one-way ANOVA followed by Tukey's multiple comparison test

killing activity [57]. Further, consistent with the findings obtained in vitro (Fig. 3d and e), the combined treatment of the aptamer and antibody significantly increased the levels of both IL-2 and IFN- $\gamma$ compared with each single treatment, as assessed by RT-qPCR on tumor samples (Fig. 5f).
The 4 T1 tumor is highly metastatic, with lungs as the principal target organ $[48,53,58]$, and depletion of $\mathrm{CD} 8+\mathrm{T}$ cells induces metastases formation in this model [52]. Notably, large metastatic foci were present in the lungs from the Ctrl group, whereas they were significantly reduced in those treated with Gint4.T or anti- 

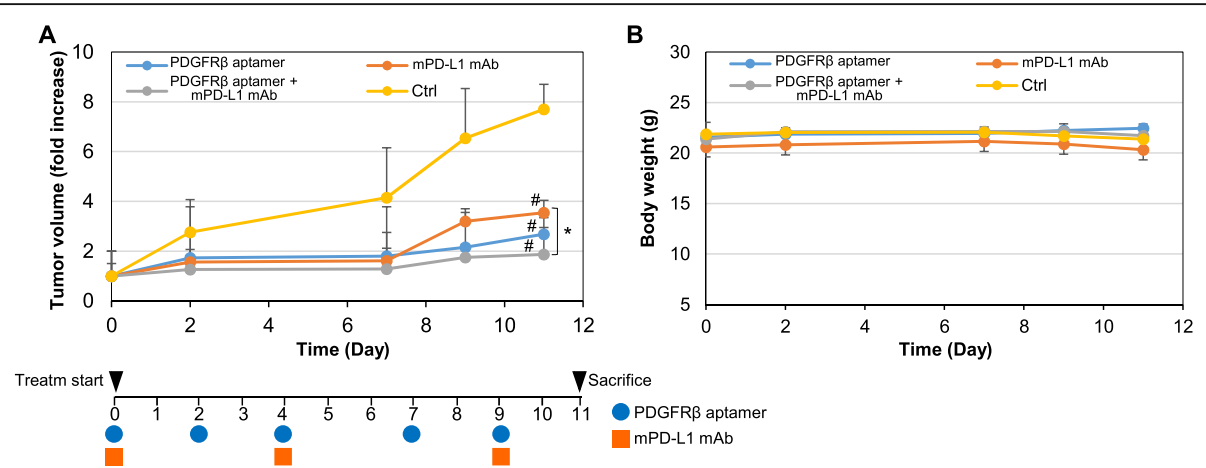

Sacrifice
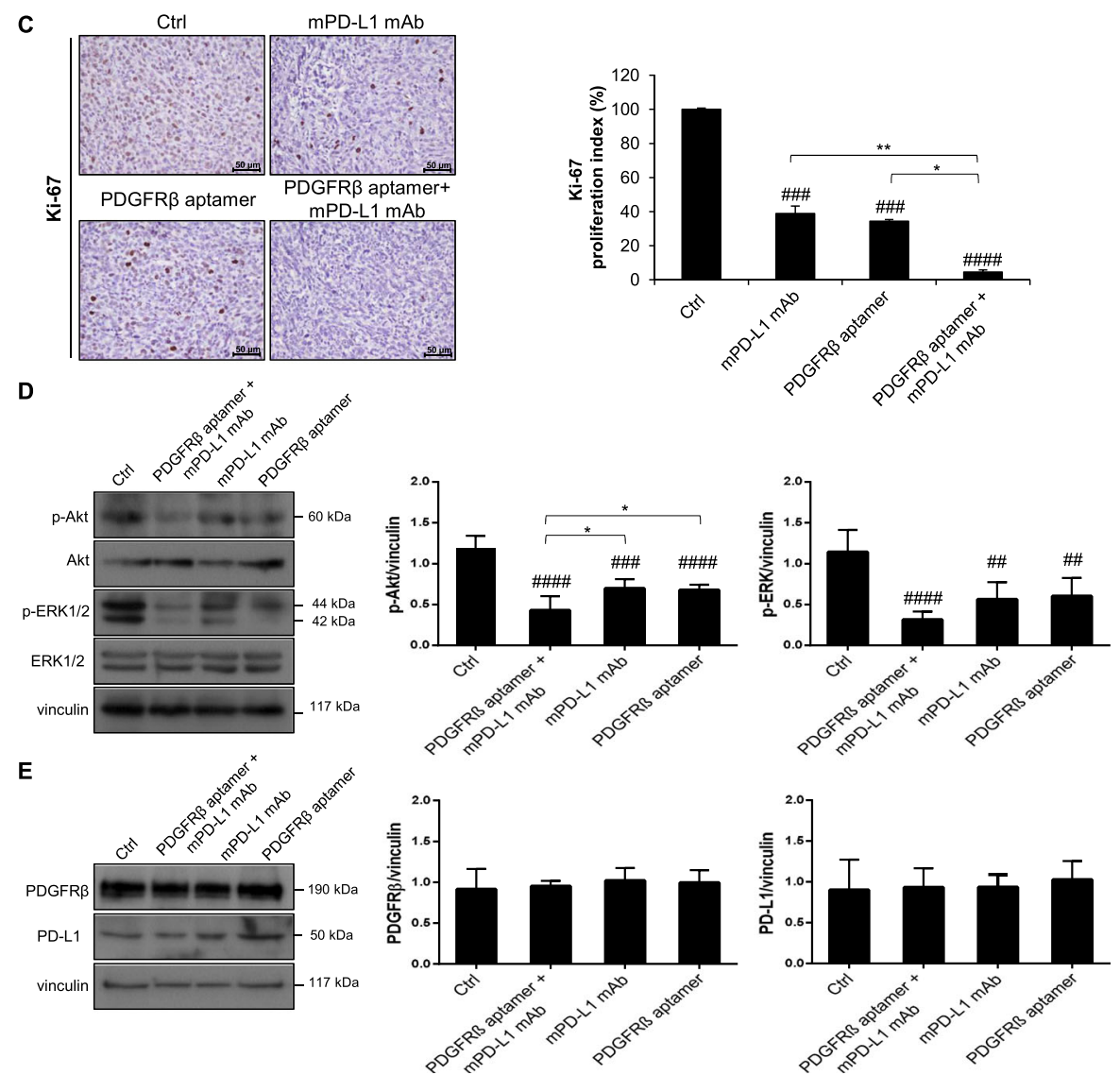

Fig. 4 Effects of combinatorial treatments on tumor growth. a Mice bearing $4 \mathrm{~T} 1$ orthotopic xenografts were injected intravenously with PDGFR Gint4.T aptamer (at day 0, 2, 4, 7 and 9) and intraperitoneally with anti-mPD-L1 mAb (at day 0, 4 and 9), alone and in combination. Mice treated with Scr aptamer were used as the control group (Ctrl). Tumor growth was monitored by calipers over time and experimental raw data (expressed as fold increase) were interpolated with no curve fitting or regression analysis. Treatment schedule is shown. Day 0 marks the start of treatments. ${ }^{\#} P<0.05$ relative to Ctrl; one-way ANOVA followed by Tukey's multiple comparison test; ${ }^{*} P<0.05$. b Mice body weight was measured at the indicated days and the mean weight of each group is shown. $\mathbf{a}-\mathbf{b}$ The mean \pm SEM $(n=5)$ were calculated for all the groups. c Shown are images from one representative tumor sample for each treatment group stained with Ki-67 antibody. Ki-67 proliferation index was calculated as percentage of Ki-67 positive cells/total cell count for 5 randomly selected $40 \times$ microscopic fields considering the Ctrl-group as 100\%. Magnification $40 \times$, scale bar $=50 \mu \mathrm{m}$. $\mathbf{d}$-e Left, lysates from recovered tumors were immunoblotted with the indicated antibodies. Equal loading was confirmed by immunoblot with anti-vinculin antibody. One representative tumor sample per group is shown. Molecular weights of indicated proteins are reported. Middle and Right, quantification of immunoblot analysis for p-Akt, p-ERK1/2, PDGFRß and PD-L1 normalized to the loading control vinculin. Bars depict mean \pm SD (five mice for each group). c-e ${ }^{\# \# \# ~} P<0.0001,{ }^{\# \# \#} P<0.001, \# \#<0.01$ relative to Ctrl; ${ }^{* *} P<0.01$; * $P<0.05$; one-way ANOVA followed by Tukey's multiple comparison test 


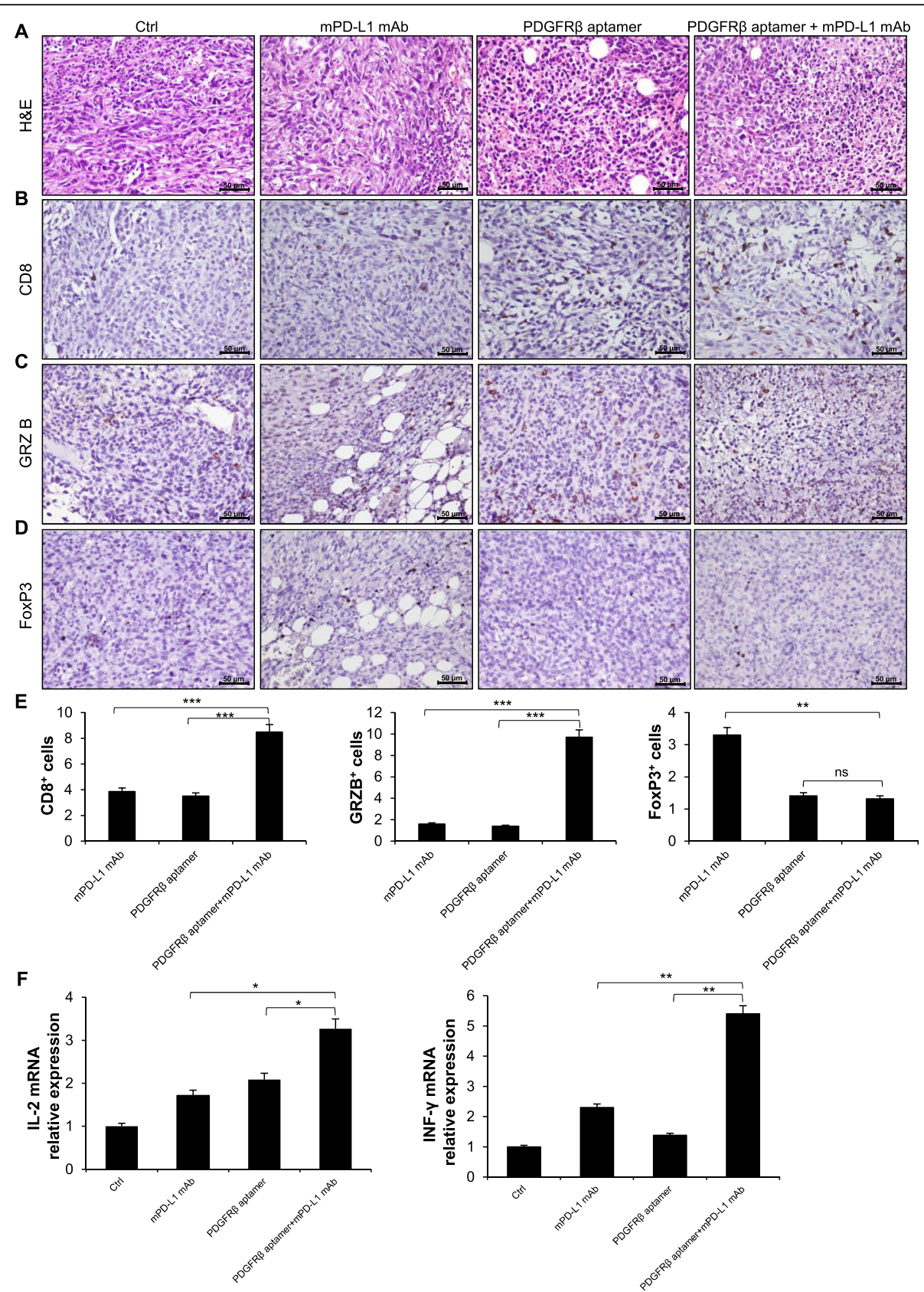

Fig. 5 Effects of combinatorial treatments on tumor-infiltrating lymphocytes. Shown are images from one representative tumor sample for each treatment group stained for H\&E (a) and immunostained with anti-CD8 (b), anti-GRZB (c) and anti-FoxP3 (d) antibodies. Magnification $40 \times$, scale bar $=50 \mu \mathrm{m}$. e CD8+, GRZB + and FoxP3+ cell counts in the tumors of anti-mPD-L1, PDGFRß aptamer and PDGFRß aptamer plus anti-mPD-L1 groups expressed relative to cell counts in Ctrl-tumors. $\mathbf{f}$ RNA extracted from recovered tumors was analyzed by RT-qPCR for the IL-2 and INF- $\gamma$ genes and mRNA relative expression was reported. Bars depict mean \pm SD $(n=4)$. e-f ${ }^{* * *} P<0.001 ;{ }^{* *} P<0.01,{ }^{*} P<0.05$; one-way ANOVA followed by Tukey's multiple comparison test

$\mathrm{mPD}-\mathrm{L} 1 \mathrm{mAb}$ and almost undetectable in the lungs of aptamer/mAb-treated mice (Fig. 6).

\section{Discussion}

Although immunotherapy approaches based on immune checkpoint inhibitors such as anti-PD-1 or anti-PD-L1 antibodies have shown convincing results in multiple cancers, they are active in only a minority of patients [59]. Thus, multiple combination approaches currently aim to improve the efficacy of PD-1/PD-L1 blockade counteracting the immunosuppressive effect of the TME and avoiding the occurrence of therapeutic resistance. However, this often requires the use of cytotoxic chemotherapy, as in the case of metastatic TNBC, which may 


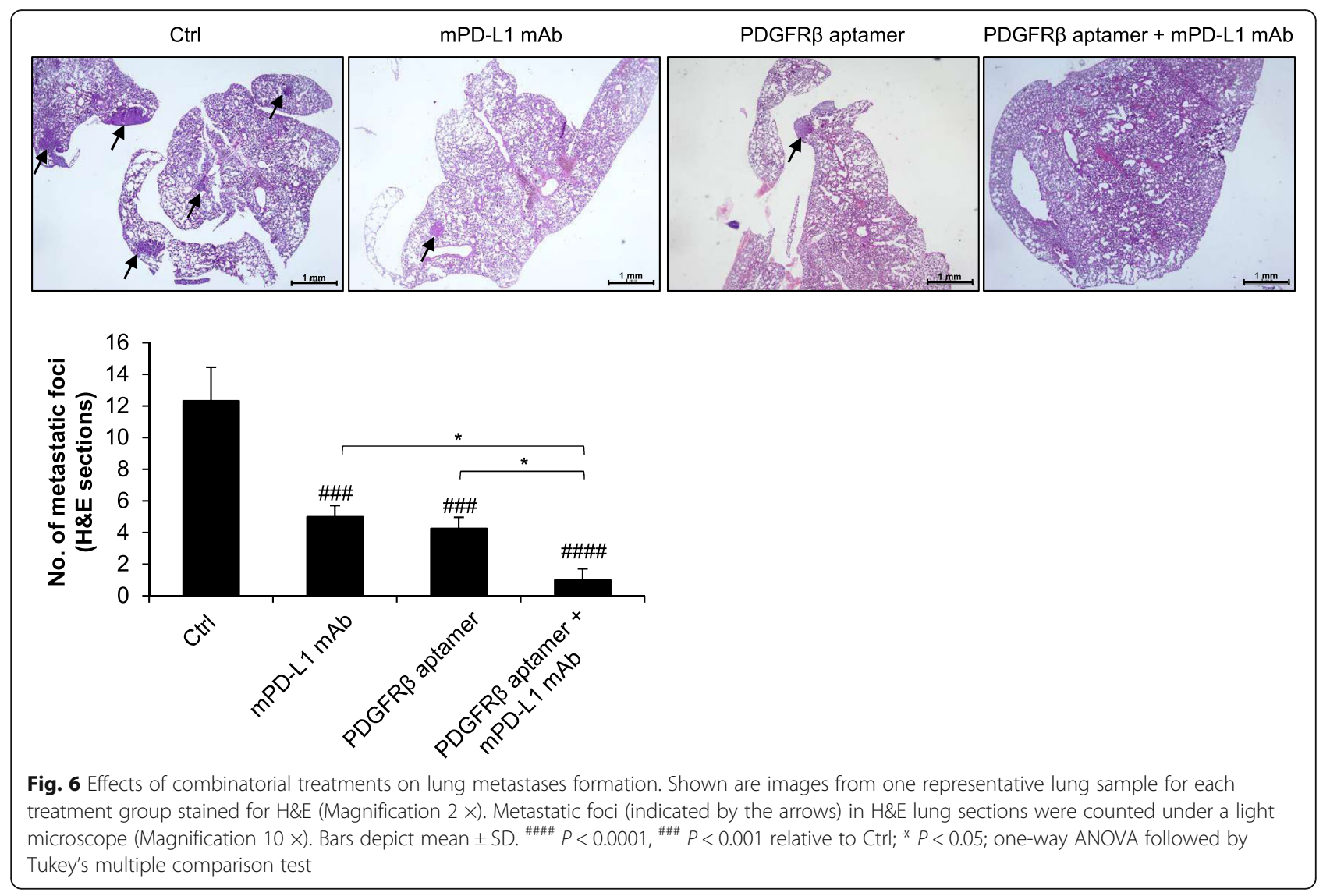

cause negative systemic side effects. In order to address this shortcoming, the ideal anti-cancer treatment alongside immunotherapy should include tumor-specific targeting agents that exhibit significant therapeutic effects and high tissue penetration. In that regards, one of the latest trends in oncotherapy is the use of aptamers, representing one of the most promising compounds able to specifically target tumor markers [60-62]. They are single-stranded oligonucleotides that, resembling antibodies, utilize their tridimensional shape for target recognition [63, 64]. Active tumor targeting by aptamers, while preserving affinity and specificity similar to mAbs, presents several advantages over them, including smaller size, higher stability, cheaper cost for synthesis, minimal inter-batch variability and lack of immunogenicity [61, 65-67]. While aptamers are generally functionalized with cytotoxic payloads for cancer therapy, it has been also demonstrated their ability to be antagonistic agents independent of drug conjugation, exerting significant potential as anti-cancer therapeutics $[31,37,68]$. PDGFR $\beta$ has been shown as an ideal target for antagonistic therapy development in aggressive human cancers, including TNBC [23-31]. Herein, the PDGFR $\beta$ antagonist Gint4.T aptamer [22, 31, 35] strengthens anti-PD-L1 antibody therapeutic efficacy in both human and murine TNBC cell cultures and in a well-established mouse model for TNBC. Our results clearly show that there are multiple mechanisms through which PDGFR $\beta$ aptamer could potentiate anti-PD-L1 antibody effects. It indeed causes inhibition of tumor growth and metastases formation by a direct effect on tumor cells, and also augments tumor immunity, which might secondarily facilitate the antitumor activity of anti-PD-L1 antibodies. The mechanism of action of the Gint4.T aptamer as both high specific targeting agent of human PDGFR $\beta$ expressed on the surface of tumor cells and inhibitor of receptor activation and downstream dependent ERK1/2 and PI3K/Akt signaling pathways in GBM [35, 36, 42] and TNBC [31], has been previously clarified and reported in literature and here confirmed for the first time in murine cells. Indeed, we show that Gint4.T is able to bind to murine PDGFR $\beta$-positive cells thus hampering cell growth and migration. Accordingly, a strong reduction of tumor growth and lung metastases was observed in orthotopic 4 T1 mouse xenografts in syngeneic BALB/c mice, which was accompanied by a strong inhibition of both ERK1/2 and Akt signaling molecules in tumor samples. Also, we suggest that Gint4.T acts on immune populations causing both the depletion of Treg cells and the increase of $\mathrm{CD}^{+} \mathrm{T}$ cells tumor infiltration together with an increase 
of GRZB, thus heightening the antibody-dependent antitumor immunity. Consistently, in both in vitro human and murine co-cultures of TNBC cells and lymphocytes and in vivo $4 \mathrm{~T} 1$ xenograft model, the aptamer potentiates the anti-PD-L1 antibody-induced $\mathrm{T}$ cell stimulation [46], as demonstrated by the increase in the levels of IL2 and IFN- $\gamma$ cytokines. Accordingly, it has been shown that PDGF may act directly on certain lymphocyte subsets $[47,69,70]$. Further, the increase of Tregs in breast tumors correlates with an invasive phenotype and a poor prognosis, and aggressive TNBC is considerably associated with high expression of Tregs [71]. Notably, small molecules inhibitors of PDGFR $\beta$ have been reported to augment tumor immunity by depletion of FoxP3expressing Tregs and increase in $\mathrm{CD}^{+} \mathrm{T}$ cells in humans and advanced tumor-bearing mice [72-74].

Tumor development and progression are promoted by the establishment of a favorable TME, including PDGF $\mathrm{R} \beta$-positive mesenchymal stem cells (MSCs), tumorassociated fibroblasts, angiogenic endothelial cells, and infiltrating immune cells, through a cytokine network [75-77]. It is likely that, by acting on these cell components, Gint4.T modifies TME to ultimately potentiate the anti-PD-L1 responses. At this regard, we previously showed that Gint4.T aptamer binds to and inhibits PDGFR $\beta$ on BM-MSCs, thus hampering their homing into TNBC TME and consequently counteracting bone marrow-derived MSCs role to enhance lung metastases formation [22]. Also, we showed that the aptamer is able to transcytose the blood-brain barrier (BBB), by binding to PDGFR $\beta$ highly expressed on endothelial cells of vessels that vascularize the tumor [36]. We could thus speculate that the aptamer may interfere with tumor vessels formation thereby inhibiting tumor growth and metastatic potential. Furthermore, because the preferential expression of PDGFR $\beta$ by cancer cells with stem-like characteristics and/or that have undergone epithelialmesenchymal transition (EMT) [31, 61], it would be interesting to assess whether the proposed aptamer-based strategy results in reducing the proportion of mesenchymal, stem-like cells. In that case it would also counteract the recently evidenced detrimental effects of chemotherapy on tumor relapse and metastasis promotion due to induction of EMT and stemness phenotype [78].

These findings provide rationale for the combined therapeutic targeting of PDGFR $\beta$ and PD-L1 in TNBC, where immune-checkpoint blockade as single therapy has met limited success so far.

Further, they lay the ground to construct a new bispecific immunoconjugate, made up of anti-PD-L1 antibody covalently linked to Gint4.T aptamer, thus optimizing the efficacy of the combination therapy by increasing their co-targeting at the tumor site while dispensing lower doses of either single agents and overcoming the limits related to the rapid clearance of the aptamers. We previously developed three different bispecific conjugates consisting of EGFR aptamer linked to either anti-HER2, anti-PD-L1 or anti-CTLA-4 mAbs [38, 39]. By this strategy the advantages of Gint4.T aptamer (nuclease resistance, rapid tumor uptake, durable tumor retention [31] and anti-PD-L1 antibodies (longer half-life in circulation, immunomodulatory activity) could be combined in one single molecule with improved therapeutic effectiveness and pharmacokinetic/pharmacodynamic properties over the parental moieties. At this regard, it would be intriguing to assess whether the conjugation of the PDGFR $\beta$ aptamer with a fragment smaller than the entire antibody, such as a Fab lacking the Fc region, preserves the aptamer's ability to deliver the antibody cargo through the BBB. In such a case the construct could exert therapeutic benefit on brain tumors or brain metastases of breast cancer, while avoiding the side effects previously seen with antibodies entering the brain [79], thanks to the lower dose of the antibody dispensed in a conjugated form.

It is still debated whether BRCA1- and BRCA2deficiency, which causes genomic instability and increased tumor burden, could increase immunosensitivity in breast cancer and predict clinical benefit from immunotherapy $[80,81]$. In the present study, we used two BRCA1- and BRCA2-proficient cell lines [82, 83]. Upcoming studies with a larger number of TNBC cell lines, either wild-type or mutated for the BRCA genes, will be helpful to answer whether BRCA1- and BRCA2mutations might influence our combined aptamerimmunotherapy.

\section{Conclusions}

PDGFR $\beta$ inhibition by a high efficacious nucleaseresistant aptamer favors antitumor immunity and potentiates anti-PD-L1 antibody responses on TNBC growth and lung metastases formation. We first propose that combining PDGFR $\beta$ blockade with anti-PD-L1 immune checkpoint inhibitors may be a viable therapeutic approach for triple-negative breast cancer.

\section{Supplementary information}

Supplementary information accompanies this paper at https://doi.org/10. 1186/s13046-020-01694-9.

Additional file 1: Figure S1. PDGFRß Gint4.T aptamer specifically binds to human and mouse lymphocytes. Cell-ELISA assays on activated human (A) and mouse (B) lymphocytes with 5'-biotinylated PDGF R $\beta$ Gint4.T aptamer at the indicated concentrations. The background binding value for 5'-biotinylated Scr aptamer was subtracted from each data point. Data shown are mean \pm SD $(n=3)$. Figure S2. Similarity of human and mouse PDGFR $\boldsymbol{\beta}$. (A) The dotplot matrix shows the sequence similarity between human and mouse PDGFR $\beta$ protein obtained by using Dotmatcher tool (EMBOSS) with a scoring matrix Blosum 62 and 
a threshold line of 13. (B) NIH3T3 cells were mock-treated or serumstarved (ss) and then left untreated (-) or stimulated with PDGF-BB, as indicated. Cell lysates were immunoblotted with the indicated antibodies. Molecular weights of indicated proteins are reported. Figure S3. PDGF $\mathbf{R} \boldsymbol{\beta}$ and PD-L1 immunohistochemical expression. Shown are images from one representative tumor for each treatment group immunostained with anti-PDGFRß (A) and anti-PDL1 (B) antibodies. Magnification $40 \times$, scale bar $=50 \mu \mathrm{m}$.

\section{Abbreviations}

2'F-Py: 2'Fluoro-pyrimidines; ATCC: American Type Culture Collection; DAPI: 4',6-Diamidino- 2-phenylindole; BB: Binding buffer; BBB: Blood-brain barrier; DPBS: Dulbecco's phosphate-buffered saline; BM-MSCs: Bone marrowderived mesenchymal stem cells; HER2: Epidermal growth factor receptor 2; EMT: Epithelial-mesenchymal transition; ERK1/2: Extracellular-signal regulated kinase 1/2; FBS: Fetal bovine serum; GBM: Glioblastoma; H\&E: Hematoxylin and eosin; hPBMCs: Human peripheral blood mononuclear cells; IFNү: Interferon gamma; IL-2: Interleukin-2; LDH: Lactate dehydrogenase; mAbs: Monoclonal antibodies; MSCs : Mesenchymal stem cells; PDGF RB: Platelet-derived growth factor receptor $\beta$; PD-L1: Programmed cell deathligand 1; PI3K: Phosphatidyl-3-kinase; RPMI-1640: Roswell Park Memorial Institute-1640 medium; RT: Room temperature; RTKs: Receptor tyrosine kinases; RT-qPCR: Reverse transcription quantitative polymerase chain reaction; TILs: Tumor-infiltrating lymphocytes; TME: Tumor microenvironment: TNBC: Triple-negative breast cancer

\section{Acknowledgements}

Not applicable.

\section{Authors' contributions}

SC, MP, LA performed most of the experiments and assisted with manuscript preparation. SE and AZ performed the animal experiments. FC and $M C$ performed the immunohistochemical staining. SC, MP, LA, FC, MC, MDB, MF, $A Z, C D L$ and $L C$ analyzed data. IVU provided reagents. MF, CDL and AZ edited the manuscript. LC and CDL supervised the study. LC conceived and designed the study, was responsible for funding and wrote the manuscript. All authors read and approved the final manuscript.

\section{Funding}

This work was supported by Fondazione AIRC per la Ricerca sul Cancro (IG 23052) to LC.

\section{Availability of data and materials}

All data generated or analyzed during in this study are included in this published article.

\section{Ethics approval and consent to participate}

All animal experimental procedures were performed in compliance with national regulations on the protection of animals used for scientific purposes (Italian decree n. 26 dated 04/03/2014 acknowledging European Directive 2010/63/ EU) with the ARRIVE guidelines and with the principle of the "3Rs" (Replacement, Reduction and Refinement). Italian Ministry of Health permission 932/2018-PR 12/12/2018.

\section{Consent for publication}

Not applicable.

\section{Competing interests}

The authors declare no conflicts of interest.

\section{Author details}

${ }^{1}$ Institute of Experimental Endocrinology and Oncology "Gaetano Salvatore", CNR, Via S. Pansini 5, 80131 Naples, Italy. 'Department of Molecular Medicine and Medical Biotechnology, University of Naples "Federico II", Via Pansini 5, 80131 Naples, Italy. ${ }^{3}$ Ceinge-Biotecnologie Avanzate s.c.a.r.l., via Gaetano Salvatore 486, 80145 Naples, Italy. ${ }^{4}$ Pathology Unit, Istituto Nazionale Tumori-IRCCS-Fondazione G. Pascale, Naples, Italy. ${ }^{5}$ Group of Experimental Biotherapy and Diagnostic, Institute for Regenerative Medicine, Sechenov First Moscow State Medical University, Moscow 119991, Russia. Institute of Biostructure and Bioimaging, CNR, Via T. De Amicis 95, 80145 Naples, Italy.
Received: 29 July 2020 Accepted: 28 August 2020

Published online: 07 September 2020

\section{References}

1. Newman LA, Reis-Filho JS, Morrow M, Carey LA, King TA. The 2014 Society of Surgical Oncology Susan G. Komen for the cure symposium: triplenegative breast cancer. Ann Surg Oncol. 2015;22:874-82.

2. Dent R, Trudeau M, Pritchard Kl, Hanna WM, Kahn HK, Sawka CA, et al. Triple-negative breast cancer: clinical features and patterns of recurrence. Clin Cancer Res. 2007;13:4429-34.

3. Gadi VK, Davidson NE. Practical approach to triple-negative breast cancer. J Oncol Pract. 2017;13:293-300.

4. Echeverria GV, Ge Z, Seth S, Zhang X, Jeter-Jones S, Zhou X, et al. Resistance to neoadjuvant chemotherapy in triple-negative breast cancer mediated by a reversible drug-tolerant state. Sci Transl Med. 2019;11:eaav0936.

5. Garrido-Castro AC, Lin NU, Polyak K. Insights into molecular classifications of triple-negative breast cancer: improving patient selection for treatment. Cancer Discov. 2019;9:176-98.

6. Keung MY, Wu Y, Badar F, Vadgama JV. Response of breast cancer cells to PARP inhibitors is independent of BRCA status. J Clin Med. 2020;9:940.

7. Cortés J, André F, Gonçalves A, Kümmel S, Martín M, Schmid P, et al. IMpassion132 phase III trial: atezolizumab and chemotherapy in early relapsing metastatic triple-negative breast cancer. Future Oncol. 2019;15:1951-61.

8. Marra A, Viale G, Curigliano G. Recent advances in triple negative breast cancer: the immunotherapy era. BMC Med. 2019;17:90.

9. Gil Del Alcazar CR, Huh SJ, Ekram MB, Trinh A, Liu LL, Beca F, et al. Immune escape in breast Cancer during in situ to invasive carcinoma transition. Cancer Discov. 2017;7:1098-115.

10. Bianchini G, Balko JM, Mayer IA, Sanders ME, Gianni L. Triple-negative breast cancer: challenges and opportunities of a heterogeneous disease. Nat Rev Clin Oncol. 2016;13:674-90.

11. Stenger M. Atezolizumab combo approved for PD-L1-positive TNBC. Cancer Discov. 2019;9:OF2.

12. Page DB, Bear H, Prabhakaran S, Gatti-Mays ME, Thomas A, Cobain E, et al. Two may be better than one: PD-1/PD-L1 blockade combination approaches in metastatic breast cancer. NPJ Breast Cancer. 2019;5:34.

13. Adams S, Gatti-Mays ME, Kalinsky K, Korde LA, Sharon E, Amiri-Kordestani L, et al. Current landscape of immunotherapy in breast cancer: a review. JAMA Oncol. 2019. https://doi.org/10.1001/jamaoncol.2018.7147.

14. García-Aranda M, Redondo M. Targeting protein kinases to enhance the response to anti-PD-1/PD-L1 immunotherapy. Int J Mol Sci. 2019;20:2296.

15. Seifert AM, Zeng S, Zhang JQ, Kim TS, Cohen NA, Beckman MJ, et al. PD-1/ PD-L1 blockade enhances T-cell activity and antitumor efficacy of imatinib in gastrointestinal stromal tumors. Clin Cancer Res. 2017;23:454-65.

16. Palakurthi S, Kuraguchi M, Zacharek SJ, Zudaire E, Huang W, Bonal DM, et al. The combined effect of FGFR inhibition and PD-1 blockade promotes tumor-intrinsic induction of antitumor immunity. Cancer Immunol Res. 2019:7:1457-71.

17. Napolitano S, Matrone N, Muddassir AL, Martini G, Sorokin A, De Falco V, et al. Triple blockade of EGFR, MEK and PD-L1 has antitumor activity in colorectal cancer models with constitutive activation of MAPK signaling and PD-L1 overexpression. J Exp Clin Cancer Res. 2019:38:492.

18. Kasikara C, Davra V, Calianese D, Geng K, Spires TE, Quigley M, et al. PanTAM tyrosine kinase inhibitor BMS-777607 enhances anti-PD-1 mAb efficacy in a murine model of triple-negative breast cancer. Cancer Res. 2019;79: 2669-83.

19. Fredriksson L, Li H, Eriksson U. The PDGF family: four gene products form five dimeric isoforms. Cytokine Growth Factor Rev. 2004;15:197-204.

20. Plate $\mathrm{KH}$, Breier $\mathrm{G}$, Farrell $\mathrm{CL}$, Risau W. Platelet-derived growth factor receptor-beta is induced during tumor development and upregulated during tumor progression in endothelial cells in human gliomas. Lab Investig. 1992;67:529-34

21. Bhowmick NA, Neilson EG, Moses HL. Stromal fibroblasts in cancer initiation and progression. Nature. 2004;18(432):332-7.

22. Camorani S, Hill BS, Fontanella R, Greco A, Gramanzini M, Auletta L, et al. Inhibition of bone marrow-derived Mesenchymal stem cells homing towards triple-negative breast Cancer microenvironment using an antiPDGFRß Aptamer. Theranostics. 2017;7:3595-607.

23. Kim Y, Kim E, Wu Q, Guryanova O, Hitomi M, Lathia JD, et al. Plateletderived growth factor receptors differentially inform intertumoral and intratumoral heterogeneity. Genes Dev. 2012;1(26):1247-62. 
24. Akhavan D, Pourzia AL, Nourian AA, Williams KJ, Nathanson D, Babic I, et al. De-repression of PDGFR $\beta$ transcription promotes acquired resistance to EGFR tyrosine kinase inhibitors in glioblastoma patients. Cancer Discov. 2013;3:534-47.

25. Fujino S, Miyoshi N, Ohue M, Takahashi Y, Yasui M, Hata T, et al. Plateletderived growth factor receptor- $\beta$ gene expression relates to recurrence in colorectal cancer. Oncol Rep. 2018;39:2178-84.

26. Zhang $H$, Sun J, Yan L, Zhao X. PDGF-D/PDGFR promotes tongue squamous carcinoma cell (TSCC) progression via activating p38/AKT/ERK EMT signal pathway. Biochem Biophys Res Commun. 2016;478:845-51

27. Wang F, Remke M, Bhat K, Wong ET, Zhou S, Ramaswamy V, et al. A microRNA-1280/JAG2 network comprises a novel biological target in highrisk medulloblastoma. Oncotarget. 2015;6:2709-24.

28. Cortez E, Gladh H, Braun S, Bocci M, Cordero E, Björkström NK, et al. Functional malignant cell heterogeneity in pancreatic neuroendocrine tumors revealed by targeting of PDGF-DD. Proc Natl Acad Sci U S A. 2016; 113:E864-73.

29. Steller EJ, Raats DA, Koster J, Rutten B, Govaert KM, Emmink BL, et al. PDGF RB promotes liver metastasis formation of mesenchymal-like colorectal tumor cells. Neoplasia. 2013;15:204-17.

30. Meng F, Speyer CL, Zhang B, Zhao Y, Chen W, Gorski DH, et al. PDGFRa and $\beta$ play critical roles in mediating Foxq1-driven breast cancer stemness and chemoresistance. Cancer Res. 2015;75:584-93.

31. Camorani S, Hill BS, Collina F, Gargiulo S, Napolitano M, Cantile M, et al. Targeted imaging and inhibition of triple-negative breast cancer metastases by a PDGFRß aptamer. Theranostics. 2018;8:5178-99.

32. D'Ippolito E, Plantamura I, Bongiovanni L, Casalini P, Baroni S, Piovan C, Orlandi R, et al. miR-9 and miR-200 regulate PDGFRß-mediated endothelial differentiation of tumor cells in triple-negative breast cancer. Cancer Res. 2016;76:5562-72.

33. Cristofanilli M, Morandi P, Krishnamurthy S, Reuben JM, Lee BN, Francis D, et al. Imatinib mesylate (Gleevec) in advanced breast cancer-expressing Ckit or PDGFR-beta: clinical activity and biological correlations. Ann Oncol. 2008;19:1713-9.

34. Curigliano G, Pivot X, Cortés J, Elias A, Cesari R, Khosravan R, et al. Randomized phase II study of sunitinib versus standard of care for patients with previously treated advanced triple-negative breast cancer. Breast. 2013;22:650-6.

35. Camorani S, Esposito CL, Rienzo A, Catuogno S, laboni M, Condorelli G, et al. Inhibition of receptor signaling and of glioblastoma-derived tumor growth by a novel PDGFRß aptamer. Mol Ther. 2014;22:828-41.

36. Monaco I, Camorani S, Colecchia D, Locatelli E, Calandro P, Oudin A, et al. Aptamer functionalization of Nanosystems for Glioblastoma targeting through the blood-brain barrier. J Med Chem. 2017;60:4510-6.

37. Camorani S, Crescenzi E, Gramanzini M, Fedele M, Zannetti A, Cerchia L. Aptamer-mediated impairment of EGFR-integrin av 33 complex inhibits vasculogenic mimicry and growth of triple-negative breast cancers. Sci Rep. 2017:7:46659.

38. Passariello M, Camorani S, Vetrei C, Cerchia L, De Lorenzo C. Novel human bispecific aptamer-antibody conjugates for efficient cancer cell killing. Cancers (Basel). 2019;11:1268

39. Passariello M, Camorani S, Vetrei C, Ricci S, Cerchia L, De Lorenzo C Ipilimumab and its derived EGFR aptamer-based conjugate induce efficient NK cell activation against cancer cells. Cancers (Basel). 2020;12:331.

40. Passariello M, D'Alise AM, Esposito A, Vetrei C, Froechlich G, Scarselli E, et al. Novel human anti-PD-L1 mAbs inhibit immune-independent tumor cell growth and PD-L1 associated intracellular signalling. Sci Rep. 2019;9:13125

41. Camorani S, Granata I, Collina F, Leonetti F, Cantile M, Botti G, et al. Novel aptamers selected on living cells for specific recognition of triple-negative breast cancer. iScience. 2020;23:100979.

42. Camorani S, Crescenzi E, Colecchia D, Carpentieri A, Amoresano A, Fedele $M$, et al. Aptamer targeting EGFRvIII mutant hampers its constitutive autophosphorylation and affects migration, invasion and proliferation of glioblastoma cells. Oncotarget. 2015;6:37570-87.

43. Vikas $P$, Borcherding $N$, Zhang $W$. The clinical promise of immunotherapy in triple-negative breast cancer. Cancer Manag Res. 2018;10:6823-33.

44. McCann KE, Hurvitz SA, McAndrew N. Advances in targeted therapies for triple-negative breast cancer. Drugs. 2019;79:1217-30.

45. Sasso E, D'Avino C, Passariello M, D'Alise AM, Siciliano D, Esposito ML, et al Massive parallel screening of phage libraries for the generation of repertoires of human immunomodulatory monoclonal antibodies. MAbs. 2018;10:1060-72.
46. Cembrola B, Ruzza V, Troise F, Esposito ML, Sasso E, Cafaro V, et al. Rapid affinity maturation of novel anti-PD-L1 antibodies by a fast drop of the antigen concentration and FACS selection of yeast libraries. Biomed Res Int. 2019;2019:6051870.

47. Daynes RA, Dowell T, Araneo BA. Platelet-derived growth factor is a potent biologic response modifier of T cells. J Exp Med. 1991;174:1323-33.

48. Kaur P, Nagaraja GM, Zheng H, Gizachew D, Galukande M, Krishnan S, et al. A mouse model for triple-negative breast cancer tumor-initiating cells (TNBC-TICs) exhibits similar aggressive phenotype to the human disease. BMC Cancer. 2012;12:120.

49. Liu J, Liao S, Huang Y, Samuel R, Shi T, Naxerova K, et al. PDGF-D improves drug delivery and efficacy via vascular normalization, but promotes lymphatic metastasis by activating CXCR4 in breast cancer. Clin Cancer Res. 2011;17:3638-48.

50. White R, Rusconi C, Scardino E, Wolberg A, Lawson J, Hoffman M, et al. Generation of species cross-reactive aptamers using "toggle" SELEX. Mol Ther. 2001:4:567-73.

51. Wang S, Campos J, Gallotta M, Gong M, Crain C, Naik E, Coffman RL, Guiducci C. Intratumoral injection of a CpG oligonucleotide reverts resistance to PD-1 blockade by expanding multifunctional CD8+ T cells. Proc Natl Acad Sci U S A. 2016;15(113):E7240-9.

52. Piranlioglu R, Lee E, Ouzounova M, Bollag RJ, Vinyard AH, Arbab AS, et al. Primary tumor-induced immunity eradicates disseminated tumor cells in syngeneic mouse model. Nat Commun. 2019;10:1430.

53. Park MK, Lee $\mathrm{CH}$, Lee $\mathrm{H}$. Mouse models of breast cancer in preclinical research. Lab Anim Res. 2018;34:160-5.

54. Herbst RS, Soria JC, Kowanetz M, Fine GD, Hamid O, Gordon MS, et al. Predictive correlates of response to the anti-PD-L1 antibody MPDL3280A in cancer patients. Nature. 2014;515:563-7.

55. Escors D, Gato-Cañas M, Zuazo M, Arasanz H, García-Granda MJ, Vera R, et al. The intracellular signalosome of PD-L1 in cancer cells. Signal Transduct Target Ther. 2018;3:26.

56. D'Alterio C, Buoncervello M, leranò C, Napolitano M, Portella L, Rea G, et al. Targeting CXCR4 potentiates anti-PD-1 efficacy modifying the tumor microenvironment and inhibiting neoplastic PD-1. J Exp Clin Cancer Res. 2019;38:432.

57. Plitas G, Konopacki C, Wu K, Bos PD, Morrow M, Putintseva EV, et al. Regulatory $T$ cells exhibit distinct features in human breast cancer. Immunity. 2016;45:1122-34.

58. DuPré SA, Redelman D, Hunter KW Jr. The mouse mammary carcinoma 4T1: characterization of the cellular landscape of primary tumours and metastatic tumour foci. Int J Exp Pathol. 2007;88:351-60.

59. Grasselly C, Denis M, Bourguignon A, Talhi N, Mathe D, Tourette A, et al. The antitumor activity of combinations of cytotoxic chemotherapy and immune checkpoint inhibitors is model-dependent. Front Immunol. 2018;9:2100.

60. Cerchia L. Aptamers: promising tools for cancer diagnosis and therapy. Cancers (Basel). 2018;10:132.

61. Camorani S, Crescenzi E, Fedele M, Cerchia L. Oligonucleotide aptamers against tyrosine kinase receptors: prospect for anticancer applications. Biochim Biophys Acta Rev Cancer. 2018;1869:263-77.

62. Mercier MC, Dontenwill M, Choulier L. Selection of nucleic acid aptamers targeting tumor cell-surface protein biomarkers. Cancers (Basel). 2017:9:69.

63. Adachi T, Nakamura Y. Aptamers: a review of their chemical properties and modifications for therapeutic application. Molecules. 2019;24:4229.

64. Hermann T, Patel DJ. Adaptive recognition by nucleic acid aptamers. Science. 2000:287:820-5.

65. Bauer M, Strom M, Hammond DS, Shigdar S. Anything you can do, I can do better: can Aptamers replace antibodies in clinical diagnostic applications? Molecules. 2019;24:4377.

66. Zhou J, Rossi J. Aptamers as targeted therapeutics: current potential and challenges. Nat Rev Drug Discov. 2017;16:181-202.

67. Keefe AD, Pai S, Ellington A. Aptamers as therapeutics. Nat Rev Drug Discov. 2010;9:537-50.

68. Macdonald J, Henri J, Goodman L, Xiang D, Duan W, Shigdar S. Development of a Bifunctional aptamer targeting the transferrin receptor and epithelial cell adhesion molecule (EPCAM) for the treatment of brain cancer metastases. ACS Chem Neurosci. 2017:8:777-84.

69. Demoulin JB, Montano-Almendras CP. Platelet-derived growth factors and their receptors in normal and malignant hematopoiesis. Am J Blood Res. 2012:2:44-56. 
70. Gersuk GM, Chang WC, Pattengale PK. Inhibition of human natural killer cell activity by platelet-derived growth factor. II. Membrane binding studies, effects of recombinant IFN-alpha and IL-2, and lack of effect on T cell and antibody-dependent cellular cytotoxicity. J Immunol. 1988;141:4031-8.

71. Bohling SD, Allison KH. Immunosuppressive regulatory $T$ cells are associated with aggressive breast cancer phenotypes: a potential therapeutic target. Mod Pathol. 2008;21:1527-32.

72. Rennert PD. Novel immunotherapeutic approaches to the treatment of cancer: drug development and clinical application. Cham: Springer International Publishing Switzerland; 2016.

73. Ozao-Choy J, Ma G, Kao J, Wang GX, Meseck M, Sung M, et al. The novel role of tyrosine kinase inhibitor in the reversal of immune suppression and modulation of tumor microenvironment for immune-based cancer therapies. Cancer Res. 2009;69:2514-22.

74. Tanaka A, Nishikawa H, Noguchi S, Sugiyama D, Morikawa H, Takeuchi Y, et al. Tyrosine kinase inhibitor imatinib augments tumor immunity by depleting effector regulatory T cells. J Exp Med. 2020;217:e20191009.

75. Hill BS, Sarnella A, D'Avino G, Zannetti A. Recruitment of stromal cells into tumour microenvironment promote the metastatic spread of breast cancer. Semin Cancer Biol. 2020;60:202-13.

76. Junttila MR, de Sauvage FJ. Influence of tumour micro-environment heterogeneity on therapeutic response. Nature. 2013;501:346-54.

77. Camorani S, Fedele M, Zannetti A, Cerchia L. TNBC challenge: oligonucleotide aptamers for new imaging and therapy modalities. Pharmaceuticals (Basel). 2018;11:123.

78. D'Alterio C, Scala S, Sozzi G, Roz L, Bertolini G. Paradoxical effects of chemotherapy on tumor relapse and metastasis promotion. Semin Cancer Biol. 2019;60:351-61.

79. Razpotnik R, Novak N, Čurin Šerbec V, Rajcevic U. Targeting malignant brain tumors with antibodies. Front Immunol. 2017;8:1181.

80. Germano G, Lamba S, Rospo G, Barault L, Magrì A, Maione F, et al. Inactivation of DNA repair triggers neoantigen generation and impairs tumour growth. Nature. 2017;552:116-20.

81. Wen WX, Leong CO. Association of BRCA1- and BRCA2-deficiency with mutation burden, expression of PD-L1/PD-1, immune infiltrates, and T cellinflamed signature in breast cancer. PLoS One. 2019;14:e0215381.

82. Schrörs B, Boegel S, Albrecht C, Bukur T, Bukur V, Holtsträter C, et al. Multiomics characterization of the $4 \mathrm{T1}$ murine mammary gland tumor model. Front Oncol. 2020;10:1195.

83. Elstrodt F, Hollestelle A, Nagel JH, Gorin M, Wasielewski M, van den Ouweland A, et al. BRCA1 mutation analysis of 41 human breast cancer cell lines reveals three new deleterious mutants. Cancer Res. 2006;66:41-5.

\section{Publisher's Note}

Springer Nature remains neutral with regard to jurisdictional claims in published maps and institutional affiliations.

Ready to submit your research? Choose BMC and benefit from:

- fast, convenient online submission

- thorough peer review by experienced researchers in your field

- rapid publication on acceptance

- support for research data, including large and complex data types

- gold Open Access which fosters wider collaboration and increased citations

- maximum visibility for your research: over $100 \mathrm{M}$ website views per year

At $\mathrm{BMC}$, research is always in progress.

Learn more biomedcentral.com/submissions 